\title{
BACTERIOLOGICAL PROFILE OF CHRONIC SUPPURATIVE OTITIS MEDIA AND ITS CLINICAL SIGNIFICANCE IN RURAL AREA
}

\author{
Mohit Srivastava ${ }^{1}$, Sushant Tyagi ${ }^{2}$ \\ ${ }^{1}$ Assistant Professor, Department of ENT, Saraswathi Institute of Medical Sciences, Hapur, Uttar Pradesh. \\ 2 Senior Resident, Department of ENT, Saraswathi Institute of Medical Sciences, Hapur, Uttar Pradesh.
}

ABSTRACT: BACKGROUND: Middle ear infection is one of the most common condition in ENT practice, particularly in our country. Its significance lies in its chronicity and its dreaded complications like hearing loss and cholesteatoma. Chronic suppurative Otitis media and its complications are among the most common conditions seen by both the otologist and pediatrician. Both gram positive and negative organisms are responsible for infection of the middle ear.

AIMS: The study was primarily carried out in 200 cases of C.S.O.M. to determine the bacteriological flora and to study the antibiotic sensitivity pattern of the organisms isolated on culture from discharging ears.

RESULTS: In our study Pseudomonas aeruginosa was found to be the most common isolated bacteria (40.65\% [100/246]), followed by Staphylococcus aureus (14.63\%[36/246]), Klebsiella aerogenes (11.78\%[29/246]) and Proteus Mirabilis (10.56\%[26/246]). Among the anerobic organisms Peptostreptococcus (5.69\%) was the most common followed by Propionibacterium (4.06\%) and Bacteroids spp. (3.65\%). Amikacin was the most effective antibiotic in the present study. It was effective against maximum number of strains 97(95.48\%) followed by Gentamycin 95(92.36\%) and Ciprofloxacin 93(91.30\%). Cefoperazone 88(86.42\%), Cefotaxime 78(74.54\%) and Ofloxacin 64(62.68\%).

CONCLUSION: Efficient and effective treatment of CSOM is based on the knowledge of causative micro-organisms and thus their antimicrobial sensitivity ensures proper clinical recovery and avoidance of the possible dreaded complications. It has been observed that human negligence is one of the main factor responsible for the development of antibiotic resistance. Along with the indiscriminate use of antibiotics by the treating physician the lack of compliance on part of the patient also results in emergence of resistance and thus in treatment failure. It has been frequently encountered that as soon as the symptoms subside and improvement in condition occurs, many patients stop taking antibiotics before completion of therapy and allow partially resistant microbes to flourish and become completely resistant or even multi drug resistant.

KEYWORDS: Chronic Suppurative Otitis Media, Pseudomonas Aeruginosa, Staphylococcus Aureus, Antimicrobial Sensitivity.

HOW TO CITE THIS ARTICLE: Mohit Srivastava, Sushant Tyagi. "Bacteriological Profile of Chronic Suppurative Otitis Media and its Clinical Significance in Rural Area". Journal of Evolution of Medical and Dental Sciences 2015; Vol. 4, Issue 90, November 09; Page: 15518-15521, DOI: $10.14260 /$ jemds/2015/2221.

INTRODUCTION: Chronic suppurative otitis media (CSOM) is the most common infection of the middle ear characterized by persistent or recurrent purulent discharge from the middle ear through a perforated tympanic membrane. Although it is a global disease, its incidence has remained relatively higher in developing countries and in poor socio economic group of populations.[1] CSOM was found to be a single major cause of conductive deafnes and was responsible for $60.27 \%$ of cases.[2] Due to the recurrent nature of the disease and the development of drug resistant pathogenic organisms, the control of infection poses a great therapeutic challenge. In CSOM the wide range of microrganisms both aerobic (eg: Pseudomonas aeruginosa, Escherichia coli, streptococus pyogenes, proteous mirabils, klebsiela species) and anaerobic (eg: Bacteroids, Peptostreptococus, Propioni bacterium) and fungi (eg: Candida, Aspergilus, Penicilium and Rhizopus) are asocialted.[3]

Financial or Other, Competing Interest: None.

Submission 16-10-2015, Peer Review 17-10-2015,

Acceptance 26-10-2015, Published 06-11-2015.

Corresponding Author:

Dr. Mohit Srivastava,

H. No. 1153, $2^{\text {nd }}$ Floor, Mohan Meakins Society,

Sector 5-Vasundhara, Ghaziabad-201012.

E-mail:dr.mohit141180@gmail.com

DOI: 10.14260/jemds/2015/2221.
The objective of our study was to examine the bacteriological profile and antibiotic sensitivity pattern to locally available antibiotics in CSOM.

MATERIAL AND METHODS: We conducted a prospective study for a period of 18 months (January 2014 to June 2015) in the Ear-Nose-Throat Outpatient Department of Saraswathi institute of medical sciences, Hapur (UP). Aural swabs were taken on the first day of attendance of the patients to ENT OPD before any local medication was given to the patient, using sterile cotton wool swabs and sterile ear specula especially prepared and autoclaved. The collected samples were enclosed in airtight plastic tubing and then transported to the microbiology test laboratory.

The samples were always taken before cleaning/suctioning the ear canals of the excess purulent exudates. Samples from bilaterally discharging ears were collected separately. The material was inoculated on Sheep Blood agar, Mac Conkey's agar, Chocolate agar, Robertson's Cooked meat broth for aerobic and anaerobic bacteria. The swabs were incubated for $48 \mathrm{hr}$ and $72 \mathrm{hr}$. Organisms were identified using standard procedures. ${ }^{[4]}$ Antimicrobial sensitivity testing for aerobic isolates was carried out by Kirby Bauer disc diffusion method on Muller Hinton agar. Results were interpreted in accordance with central laboratory standards institute guidelines. ${ }^{[5]}$ 
Statistical Analysis: The Statistical Package for Social Sciences (SPSS) version 16 was used for analysis. Chi-square test was used to test the statistical significance of the association between potential variables and $p$-value of $<0.05$ was considered statistically significant.

RESULTS: Among the 200CSOM cases studied, 127 were unilateral cases $(63.5 \%[127 / 200])$ while bilateral CSOM were recorded in 73patients (73/200[36.5\%]), thereby making a total of 273 ear cultures available for analysis. Their ages ranged from 8months to 74years. Fifty-two percent were children aged 8 months to 18 years. The males accounted for $57 \%(114 / 200)$ of the patients with a male to female ratio of 1.32:1.

Out of total 273 ear swabs processed microbial growth was seen in 246 (90.11\%) while 27(9.89\%) samples showed no growth. In $148(60.17 \%)$ samples mono-microbial growth was seen whereas 98(39.83\%) samples showed polymicrobial growth.

In our study Pseudomonas aeruginosa was found to be the most common isolated bacteria (40.65\%[100/246]), followed by Staphylococcus aureus 14.63\%[36/246]), Klebsiella aerogenes $(11.78 \%[29 / 246])$ and Proteus Mirabilis (10.56\%[26/246]). Among the anerobic organisms Peptostreptococcus (5.69\%) was the most common followed by Propionibacterium (4.06\%) and Bacteroids spp. (3.65\%).

DISCUSSION: Chronic Suppurative Otitis Media (C.S.O.M.) and its complications are among the most common conditions seen by the ENT Practitioner. It is a persistent disease with great risk of irreversible complications. CSOM is an important cause of preventable hearing loss particularly in the developing world.[6] and a reason of serious concern, particularly in children, because it may have long-term effects on early communication, language development, auditory processing, educational process, and physiological and cognitive development.[7] Early, microbiological diagnosis ensures prompt and effective treatment to avoid such complications. We found that the CSOM was more prevalent in first and second decade of life and accounted for $52 \%$ of the cases. High-prevalence of CSOM in children may be attributed to the fact that they are more prone to upper respiratory tract infections (URTIs). Furthermore, cold weather pre-disposes children to URTI. Along with these poor socio-economic status and unhygienic conditions and improper sanitation habits can further predispose to recurrent ear discharge.[8][9]

Predominant bacterial etiology of CSOM in our study was Pseudomonas aeruginosa seen in $40.65 \%$ of the cases followed by Staphylococcus aureus (14.63\%) and Klebsiella aerogenes(11.78\%). Pseudomonas, is the predominant cause of CSOM in tropical region does not usually inhabit the upper respiratory tract, its presence in the middle-ear cannot be ascribed to an invasion through ET and it should be considered as secondary invader gaining access to the middle-ear via defect in TM.[10]

Proteus mirabilis was seen in $10.56 \%$ of the cases and Escherichia coli were isolated from $8.94 \%$ cases, and these findings were similar to the reports by Mansoor, et al.[11] and Poorey and lyer.[8]
Among the annerobes Peptostreptococcus was found in $5.69 \%$ cases followed by Bacteroides spp. In $3.65 \%$ and Propionibacterium (4.06\%). The role of anaerobes in CSOM is often questioned as they are mostly detected in cases with extensive cholesteatoma or granulation tissue however, it is advocated that while investigating pathogenic organisms in CSOM requests for anaerobic culture should be included and the medical therapy should be directed at the eradication of the pathogenic aerobic and anaerobic organisms.[12] There was strong evidence that mixed aerobic, anaerobic cultures characterized chronic infection suggesting a potential synergy between anaerobic and aerobic bacteria. It has been previously reported that polymicrobial infections are more pathogenic than mono-microbial infections. [13]

Antimicrobial susceptibility test (AST) was carried out for all the isolates. Amikacin was found to be most effective drug followed by gentamycin and ciprofloxacin. Studies have revealed that quinolones like CIP are safe and effective particularly against $\mathrm{S}$. aureus and Pseudomonas aeruginosa. Amikacin was the most effective antibiotic in the present study.

It was effective against maximum number of strains 97(95.48\%) followed by Gentamycin 95(92.36\%) and Ciprofloxacin 93(91.30\%). Cefoperazone 88(86.42\%), Cefotaxime $78(74.54 \%)$ and Ofloxacin $64(62.68 \%)$. It is clear that microbial profile and AST pattern of CSOM has been changing with due course of time. Geographical variation and difference in-patient population studied could be the possible factor for variability. Emergence of antimicrobial resistance is becoming more common. Indiscriminate and haphazard antibiotic use as well as negligence on patient part are the factors responsible.[14]

CONCLUSION: A thorough and precise knowledge of the etiological agents causing $\mathrm{CSOM}$ and their antimicrobial susceptibility is of essential importance for an effective and efficient treatment and thus in further prevention of both complications and development of antibiotic resistance which is is becoming more common. It has been observed that human negligence is one of the main factor responsible for the development of antibiotic resistance.

Along with the indiscriminate use of antibiotics by the treating physician the lack of compliance on part of the patient also results in emergence of resistance and thus in treatment failure. It has been frequently encountered that as soon as the symptoms subside and improvement in condition occurs, many patients stop taking antibiotics before completion of therapy and allow partially resistant microbes to flourish and become completely resistant or even multi drug resistant. Such practices should be condemned strongly and patients should be educated properly to avoid such behavior and its consequences.

\section{REFERENCES:}

1. Shaheen MM, Raquib A, Ahmad SM. Prevalence and associated socio-demographic factors of chronic suppurative otitis media among rural primary school children of Bangladesh. Int J Pediatr Otorhinolaryngol.2012; 76:1201-4.

2. Ogisi FO. Impedance screening for otitis media with effusion in Nigerian children. J.L.0.198; 102:986-8.

3. Shazia Parven SJ. Aerobic bacteriology of CSOM in a teaching hospital. Microbiol and Biotech. Res. 2012, 2(4); 108:1306-10. 
4. MacFaddin J. 3rd ed. Philadelphia: Lippincott Williams and Wilkins; 1976. Biochemical Tests for Identification of Medical Bacteria.

5. Performance Standards for Antimicrobial Susceptibility Testing. Vol. 1 No. 1, M2 A9. Vol. 1. Pennsylvania, USA: Clinical and Laboratory Standard Institute; 2007. Clinical and Laboratory Standard Institute.

6. Berman S. Otitis media in developing countries. Pediatrics. 1995;96:126-31.

7. Acuin J. Geneva: World Health Organisation; 2004. Global burden of disease due to chronic suppurative otitis media: Disease, deafness, deaths and DALYs Chronic Suppurative Otitis Media-Burden of Illness and Management Options; pp. 9-23. (Accessed August 29, 2012).

8. Poorey VK, Lyer A. Study of bacterial flora in csom and its clinical significance. Indian J Otolaryngol Head Neck Surg. 2002;54:91-5.

9. Gordon MA, Grunstein E, Burton WB. The effect of the season on otitis media with effusion resolution rates in the New York Metropolitan area. Int J Pediatr Otorhinolaryngol. 2004;68:191-5.
10. Vishvanath S, Mukhopadhyay C, Prakash R, Pillai S, Pujary K, Pujary P. Chronic suppurative otitis media: Optimizing initial antibiotic therapy in a tertiary care setup. Indian J Otolaryngol Head Neck Surg.2012;64:285-9.

11. Mansoor T, Musani MA, Khalid G, Kamal M. Pseudomonas aeruginosa in chronic suppurative otitis media: Sensitivity spectrum against various antibiotics in Karachi. J Ayub Med Coll Abbottabad.2009;21:120-3.

12. Brook I. The role of anaerobic bacteria in chronic suppurative otitis media in children: Implications for medical therapy. Anaerobe. 2008;14:297-300.

13. Brook I. Microbiology of polymicrobial abscesses and implications for therapy. J Antimicrob Chemother. 2002;50:805-10.

14. Microbiology of Chronic Suppurative Otitis Media in a Tertiary Care Setup of Uttarakhand State, India ; Rajat Prakash, Deepak Juyal, Vikrant Negi, Shekhar Pal, Shamanth Adekhandi, Munesh Sharma, Neelam Sharma ; N Am J Med Sci. 2013 April; 5(4): 282-287. doi: 10.4103/1947-2714.110436.

\begin{tabular}{|c|c|c|c|c|}
\hline \multirow{2}{*}{$\begin{array}{c}\text { ISOLATED } \\
\text { SPECIES }\end{array}$} & \multicolumn{3}{|c|}{ NUMBER OF CASES } & \multirow{2}{*}{ TOTAL } \\
\cline { 2 - 4 } & \multirow{2}{*}{ UNILATERAL CSOM } & \multicolumn{2}{|c|}{ BILATERAL CSOM } & \multirow{2}{*}{} \\
\cline { 2 - 4 } & & RIGHT EAR & LEFT EAR & $100(40.65 \%)$ \\
\hline Pseudomonas aeruginosa & 41 & 30 & 29 & $29(11.78 \%)$ \\
\hline Klebsiella aerogenes & 15 & 6 & 8 & $36(14.63 \%)$ \\
\hline Staph. Aureus & 18 & 12 & 6 & $22(8.94 \%)$ \\
\hline E.Coli & 10 & 4 & 8 & $26(10.56 \%)$ \\
\hline Proteus mirabilis & 17 & 6 & 3 & $14(5.69 \%)$ \\
\hline Peptostreptococcus & 5 & 4 & 5 & $9(3.65 \%)$ \\
\hline Bacteroides spp. & 4 & 2 & 3 & $10(4.06 \%)$ \\
\hline Propionibacterium & 5 & 3 & 2 & $27(9.89 \%)$ \\
\hline No growth & 8 & 10 & 9 & \\
\hline \multicolumn{2}{|c|}{ Table 1: Occurrence of Various Pathogenic Organisms in Patients of C.S.O.M. } \\
\hline
\end{tabular}

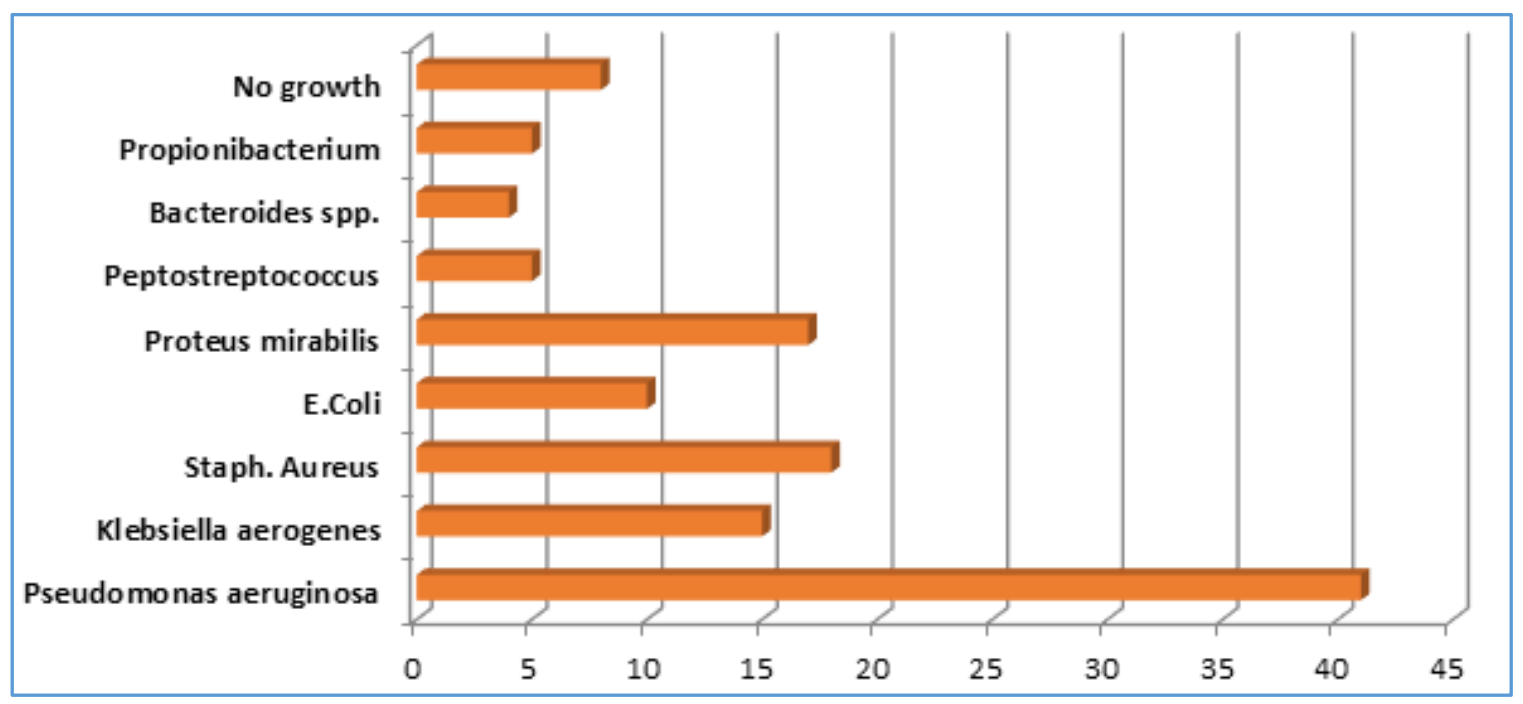

Fig. 1: Showing isolated organisms in unilateral csom. 


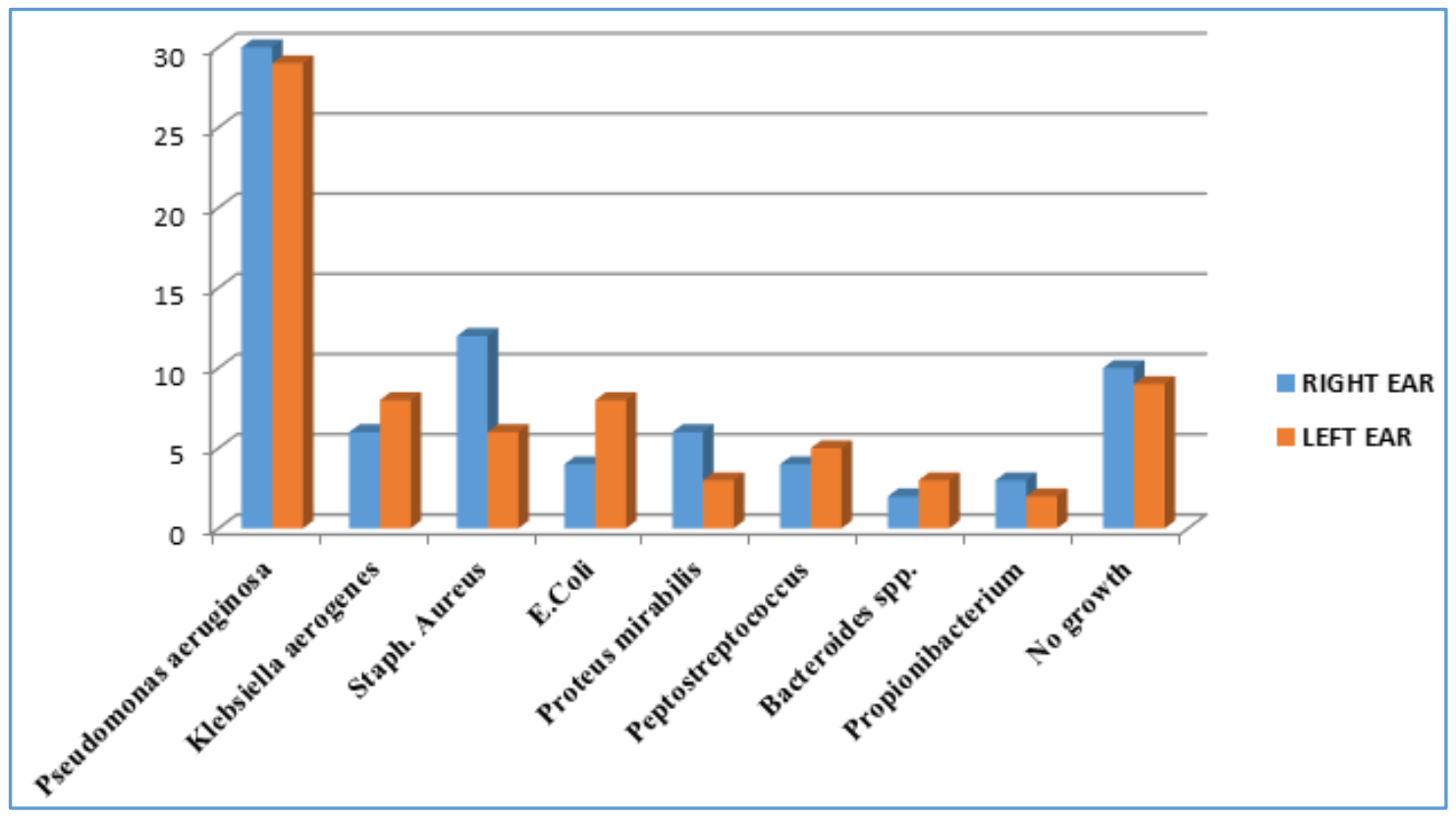

Fig. 2: Shows the incidence of microbes in each ear in cases of bilateral csom.

Antimicrobial susceptibility test (AST) was carried out for all the isolates. Amikacin was the most effective antibiotic in the present study. It was effective against maximum number of strains 97(95.48\%) followed by Gentamycin 95(92.36\%) and Ciprofloxacin 93(91.30\%). Cefoperazone 88(86.42\%), Cefotaxime 78(74.54\%) and Ofloxacin 64(62.68\%).

\begin{tabular}{|c|c|c|}
\hline Antibiotic & Susceptible Strains & Percentage \% \\
\hline Amikacin & 97 & 95.48 \\
\hline Gentamycin & 95 & 92.36 \\
\hline Ciprofloxacin & 93 & 91.30 \\
\hline Cefoperazone & 88 & 86.42 \\
\hline Cefotaxime & 78 & 74.54 \\
\hline Ofloxacin & 64 & 62.68 \\
\hline
\end{tabular}

